
FOLLOW UP

Beata Drab ${ }^{1}$, Katarina Aili ${ }^{2,3,4}$, Emma Haglund ${ }^{3,5}$, Stefan Bergman ${ }^{1,3} .{ }^{1}$ Institute of medicine, University of Gothenburg, Primary Health Care Unit, Department of Public Health and Community Medicine, Gothenburg, Sweden; ${ }^{2}$ School of Health and Welfare, Halmstad University, Halmstad, Sweden; ${ }^{3}$ RandD Spenshult, Halmstad, Sweden; ${ }^{4}$ Institute of Environmental Medicine, Karolinska Institute, Stockholm, Sweden; ${ }^{5}$ School of Business, Engineering and Science, Halmstad University, Halmstad, Sweden

Background: Chronic musculoskeletal pain (CMP) is a common cause of disability and impaired quality of life. In Sweden, chronic pain and mental illness are major causes of sick leave. But sick leave itself is also proposed as a risk factor for prolonged sick leave and disability pension.

Objectives: To study CMP and sick leave as potential risk factors for long term sick leave or disability pension in a 21 year follow up of a general population cohort.

Methods: In a cohort study, with a baseline survey in 1995, 1466 individuals aged 20-67 years were followed for 3 years and 691 for 21 years, or up to the age of 67. CMP ( $>3$ months duration) was reported on a pain mannequin. Sick leave and disability pension were self-reported. Mental health was measured by the mental health (MH) score of the SF-36 health status, and categorized into tertiaries (best, medium and worst). CMP, sick leave, and mental health at baseline, were studied as potential predictors for long term sick leave (disability pension or sick leave $>3$ months) at a 3 and 21 year follow up. Other potential predictors (socioeconomic group, education, and immigrant status) were introduced in multiple regression analyzes but did not add to the results and were removed from the final models, which were controlled for age and sex.

Results: CMP and mental health predicted long term sick leave at the 3 year follow up (OR 2.11, $\mathrm{p}=0.010$ and $\mathrm{OR} 3.52, \mathrm{p}<0.001)$. Mental health (OR 1.92, $\mathrm{p}=0.046)$, but not CMP (OR $0.77, \mathrm{p}=0.409)$, was also a predictor at the 21 year follow up. Sick leave $>3$ months, irrespectively if due to pain or not, predicted long term sick leave both at the 3 and the 21 year follow up (Table). Sick leave for $\leq 3$ months also predicted long term sick leave at both follow ups when due to pain (OR 2.70, $\mathrm{p}=0.008$ and OR 2.78, $\mathrm{p}=0.012$ ), but not when due to other causes (OR $1.52, \mathrm{p}=0.212$ and $\mathrm{OR} 1.17, \mathrm{p}=0.606$ )

\begin{tabular}{|c|c|c|c|c|}
\hline & \multicolumn{2}{|c|}{$\begin{array}{l}\text { Long term sick-leave or } \\
\text { disability pension at } 3 \text { year } \\
\text { follow up vs. all other }\end{array}$} & \multicolumn{2}{|c|}{$\begin{array}{l}\text { Long term sick-leave or } \\
\text { disability pension at } 21 \text { year } \\
\text { follow up vs. all other }\end{array}$} \\
\hline & $\mathrm{OR}(95 \% \mathrm{Cl})$ & $\mathrm{p}$ & $\mathrm{OR}(95 \% \mathrm{Cl})$ & $p$ \\
\hline \multicolumn{5}{|l|}{ Sick leave 1995} \\
\hline No sick leave & 1 & & 1 & \\
\hline$\leq 3$ months due to pain & $2.70(1.29-5.63)$ & 0.008 & $2.78(1.26-6.14)$ & 0.012 \\
\hline$>3$ months due to pain & $7.81(2.66-22.94)$ & $<0.001$ & $15.60(2.28-106.50)$ & 0.005 \\
\hline$\leq 3$ months other cause & $1.52(0.79-2.96)$ & 0.212 & $1.17(0.65-2.09)$ & 0.606 \\
\hline$>3$ months other cause & $4.60(1.13-18.60)$ & 0.033 & $9.22(1.21-70.30)$ & 0.032 \\
\hline \multicolumn{5}{|l|}{ CMP in 1995} \\
\hline no & 1 & & 1 & \\
\hline yes & $2.11(1.19-3.71)$ & 0.010 & $0.77(0.42-1.42)$ & 0.409 \\
\hline \multicolumn{5}{|l|}{ SF-36 MH 1995} \\
\hline Best & 1 & & 1 & \\
\hline Medium & $1.63(0.73-3.61)$ & 0.231 & $1.63(0.87-3.09)$ & 0.128 \\
\hline Worst & $3.52(1.75-7.10)$ & $<0.001$ & $1.92(1.01-3.66)$ & 0.046 \\
\hline
\end{tabular}

Conclusion: Sick leave and especially sick leave due to pain predicted long term sick leave up to 21 years later, independently of pain status or mental health at baseline. It is thus important to early identify individuals at risk and minimize sick leave by providing proper rehabilitation.

Disclosure of Interests: None declared

DOI: 10.1136/annrheumdis-2019-eular.2021

\section{OP0098 SUICIDAL BEHAVIOUR IN FIBROMYALGIA PATIENTS: META-ANALYSIS AND SYSTEMATIC REVIEW OF THE LITERATURE}

Mohammad Adawi ${ }^{1,2}$, Nicola Luigi Bragazzi ${ }^{3}$, Dennis Mcgonagle ${ }^{4}$, Abdulla Watad $^{5}$, Howard Amital ${ }^{5} .{ }^{1}$ Bar-llan University, Ramat Gan, Israel; ${ }^{2}$ Poria medical center, Poria, Israel; ${ }^{3}$ University of Genoa, Genoa, Italy, Postgraduate School of Public Health, Department of Health Sciences (DISSAL), Genoa, Italy; ${ }^{4}$ University of Leeds, Section of Musculoskeletal Disease, Leeds, United Kingdom; ${ }^{5}$ Sheba Medical Center, Internal Medicine B, Tel-aviv, Israel

Background: Suicide is a leading cause of death worldwide, with approximately 800,000 people dying every year. Fibromyalgia is the second most common rheumatic disease, affecting $0.2-6.6 \%$ of the population worldwide and fibromyalgia patients suffer from anxiety and depression, which are known to be risk factors for suicidal behaviour. Suicidality related to chronic pain conditions has been studied, but there is a dearth of information specifically concerning suicidality among patients with fibromyalgia.

Objectives: To investigate the risk of suicidal ideation and attempts in patients with fibromyalgia.

Methods: A systematic review and meta-analysis was conducted and reported according to the "Preferred Reporting Items for Systematic reviews and Metaanalyses" (PRISMA) standards. Also, the gray literature was extensively searched.

Results: Thirteen studies were included in the present systematic review and meta-analysis, of 394,087 fibromyalgia patients. Sample size ranged from 44 to 199,739 subjects: mean age ranged from 45.8 to 54.5 years while female percentage went from $17.1 \%$ to $100.0 \%$. Fibromyalgia diagnosis was based on the 1999 ACR criteria and $2010 \mathrm{ACR}$ criteria in one study. The overall suicide ideation prevalence was $29.57 \%(95 \% \mathrm{Cl} 1.84-72.07)$, with an OR 9.12 of $(95 \% \mathrm{Cl} 1.42-58.77)$, ranging from $2.34(95 \% \mathrm{Cl} 1.49-3.66)$ to $26.89(95 \% \mathrm{Cl} 5.72-126.42)$. Pooled suicide attempt prevalence was $5.69 \%$ [ $95 \% \mathrm{Cl} 1.26-31.34]$, with an OR of 3.12 [95\% $\mathrm{Cl} 1.37-7.12]$. Suicide risk was higher with respect to the general population with an OR of $36.77(95 \% \mathrm{Cl} 15.55-96.94)$, as well as suicide events with an HR of 1.38 $(95 \% \mathrm{Cl} 1.17-1.71)$. Determinants of suicidality were found to be: employment status, disease severity, obesity and drug dependence, chronic pain and co-morbidities, in particular depression, anxiety, poor sleep, and global mental health.

Conclusion: Fibromyalgia patients are particularly prone to suicide, in terms of ideation, attempt, risk and events, warranting a pre-emptive screening of their mental health status. However, in some cases, after adjusting for psychiatric conditions, the threshold of statistical significance was not achieved. Given the few studies available, the high amount of heterogeneity and the evidence of publications bias, further high-quality studies should be conducted.

Table 1. The main findings of the systematic review and meta-analysis on suicidality among fibromyalgia patients.

\begin{tabular}{ll}
\hline Variable & Results \\
\hline Suicide & Overall suicide ideation prevalence $29.57 \%[95 \% \mathrm{Cl} 1.84-72.07] ; 1.08-54.6 \%$ \\
ideation & Passive suicide ideation prevalence $39.86 \%[95 \% \mathrm{Cl} 35.14-44.72] ; 39.7-$ \\
& $41.0 \%$ \\
& Active suicide ideation prevalence $9.00 \%[95 \% \mathrm{Cl} 6.44-12.16] ; 8.3-13.6 \%$ \\
& OR $9.12[95 \% \mathrm{Cl} 1.42-58.77] ; 2.34[95 \% \mathrm{Cl} 1.49-3.66]-26.89[95 \% \mathrm{Cl} 5.72-$ \\
& $126.42]$ \\
& OR $7.60[95 \% \mathrm{Cl} 0.72-80.17]$ adjusted for psychiatric conditions \\
Suicide & Prevalence $5.69 \%[95 \% \mathrm{Cl} 1.26-31.34] ; 0.38-16.67 \%$ \\
attempt & OR $3.12[95 \% \mathrm{Cl} 1.37-7.12]$ \\
& OR $1.27[95 \% \mathrm{Cl} 0.54-3.01]$ adjusted for psychiatric conditions \\
Suicide risk & OR from $36.77[95 \% \mathrm{Cl} 15.55-96.94] ; 30.06[95 \% \mathrm{Cl} 10.25-100.27]$ to 48.0 \\
& [95\%Cl $12.93-178.21]$ \\
& HR $1.45[95 \% \mathrm{Cl} 1.16-1.81]$ \\
& HR $1.16[95 \% \mathrm{Cl} 0.92-1.44]$ adjusted for psychiatric conditions \\
Suicide event & HR $1.38[95 \% \mathrm{Cl} 1.17-1.71]$ \\
& Incidence $31 / 100,000$ person/years \\
& Mortality $\mathrm{OR} 3.31[95 \% \mathrm{Cl} 2.15-5.11]$ \\
& SMR $10.5[95 \% \mathrm{Cl} 4.5-20.7]$ \\
\hline
\end{tabular}

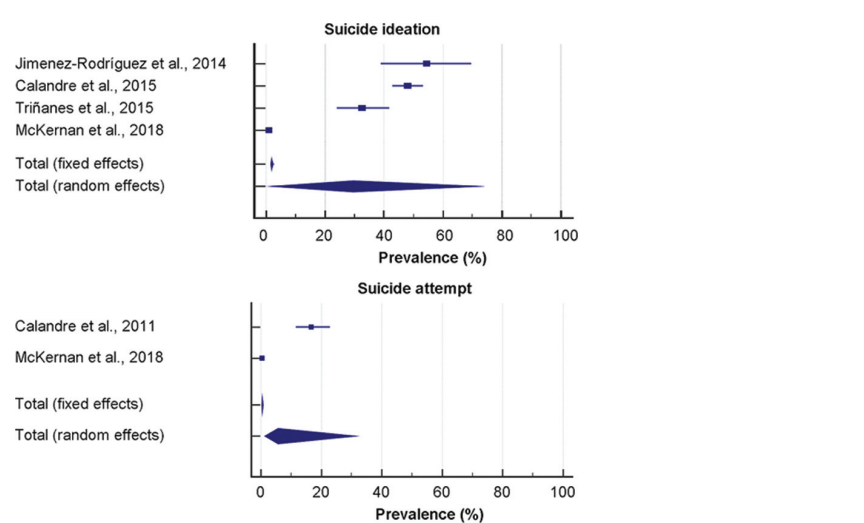

Figure 1. Forest plots of suicide ideation and attempt rates among fibromyalgia patients.

Disclosure of Interests: Mohammad Adawi: None declared, Nicola Luigi Bragazzi: None declared, Dennis McGonagle Consultant for: Lilly, Novartis UCB, Speakers bureau: Lilly, Novartis UCB, Abdulla Watad: None declared, Howard Amital Grant/research support from: Pfizer, AbbVie, Janssen, Grant/research support from: Pfizer, AbbVie, Janssen, Consultant for: Pfizer, Merck Sharp \& Dohme, Consultant for: Pfizer, Merck Sharp \& Dohme, Speakers bureau: Pfizer Merck Sharp \& Dohme, Janssen, Sanofi, Bristol-Myers Squibb, Abbvie, Neopharm, Speakers bureau: Pfizer, Merck Sharp \& Dohme, Janssen, Sanofi, BristolMyers Squibb, Abbvie, Neopharm DOI: 10.1136/annrheumdis-2019-eular.1076 